\title{
A CANONICAL PARTITION OF THE PERIODIC ORBITS OF CHAOTIC MAPS
}

BY

\author{
KATHLEEN T. ALLIGOOD ${ }^{1}$
}

\begin{abstract}
We show that the periodic orbits of an area-contracting horseshoe map can be partitioned into subsets of orbits of minimum period $k, 2 k, 4 k, 8 k$, $\ldots$, for some positive integer $k$. This partition is natural in the following sense: for any parametrized area-contracting map which forms a horseshoe, the orbits in one subset of the partition are contained in a single component of orbits in the full parameter space. Furthermore, prior to the formation of the horseshoe, this component contains attracting orbits of minimum period $2^{m} k$, for each nonnegative integer $m$.
\end{abstract}

For parametrized maps of $R^{n}$ which form horseshoes, there is a rich structure of attracting periodic points for parameter values which precede the existence of the horseshoe. However, once the horseshoe is fully formed, all periodic points are unstable. Let $f_{\lambda}: R^{n} \rightarrow R^{n}(0 \leq \lambda \leq 1)$ be a $C^{1}$ map which contracts crosssectional areas. (Precise formulations of hypotheses follow this introduction.) We assume that $f_{0}$ has either no periodic orbits or only attracting ones, and that $f_{1}$ has only unstable periodic orbits (as, for example, in the horseshoe map). A periodic point $p$ of an area-contracting map can have at most one unstable direction. Thus if $p$ is unstable, then $D f_{\lambda}^{k}(p)$ (where $k$ is the period of $p$ ) has exactly one real eigenvalue $\mu$ such that $|\mu|>1$. These periodic orbits fall into two classes: if $\mu$ is in $(1, \infty)$, we call $p$ a saddle orbit; if $\mu$ is in $(-\infty,-1)$, we call it a Möbius orbit. Franks $[\mathbf{F}]$ showed that the existence of an orbit of period $k$ at $\lambda=1$ (i.e., a periodic orbit of $f_{1}$ ) implies the existence of a sequence $\left\{q_{i}\right\}_{i \geq 0}$ of Möbius orbits at $\lambda=1$, where the period of $q_{i}$ is $2^{i} k$, for each $i \geq 0$. Here we show that the set of all the periodic orbits of $f_{0}$ and $f_{1}$ can be partitioned into disjoint subsets. Each of these subsets contains an attractor of period $k$ (for some $k \geq 1$ ) at $\lambda=0$ or a saddle of period $k$ at $\lambda=1$, and a sequence $\left\{q_{i}\right\}_{i \geq 0}$ of Möbius orbits at $\lambda=1$, where the period of $q_{i}$ is $2^{i} k$, for each $i \geq 0$. Hence the existence of an orbit at $\lambda=0$ or $\lambda=1$ implies the existence of all other orbits in the subset to which it belongs. Furthermore, this partition is a natural one in the sense that an entire subset lies in one connected component of periodic orbits in $(x, \lambda)$-space (Theorem 2).

We also examine how the unstable periodic orbits at $\lambda=1$ are related to the attractors which occur in the parameter range $0 \leq \lambda \leq 1$. In [YA] it was shown that if $f$ has either an attractor of period $k$ at $\lambda=0$ or a saddle of period $k$ at $\lambda=1$, then it must have a period-doubling cascade of attractors (i.e., $f$ has a sequence $\left\{a_{i}\right\}_{i \geq 0}$ of attractors such that the period of $a_{i}$ is $2^{i} k$, for each $i \geq 0$ ).

Received by the editors September 20, 1984 .

1980 Mathematics Subject Classification. Primary 58F13, 58F14; Secondary 34C35.

${ }^{1}$ Research was partially supported by the National Science Foundation. This paper was written while the author was visiting Michigan State University and the University of Maryland. 
Here we show that every periodic orbit at $\lambda=1$ lies on a (connected) component of orbits (in $(x, \lambda)$-space) which contains a periodic-doubling cascade of attractors (Theorem 1).

I would like to mention that although no formal index theory is used to obtain these results, the techniques used here were motivated by those in $[\mathbf{M Y}]$ and $[\mathbf{Y A}]$, which depend on the orbit (or $\varphi$-) index.

First, we specify hypotheses for $f$. Let

$$
C=\left\{\left(x_{1}, \ldots, x_{n}\right) \in R^{n}: x_{1}^{2}+\cdots+x_{n-1}^{2} \leq 1 \text { and } 0 \leq x_{n} \leq 1\right\} .
$$

We consider a $C^{1}$ map $f: C \times[0,1] \rightarrow R^{n}$ (writing $f_{\lambda}$ for $\left.f(\cdot, \lambda)\right)$ and let

$$
P=\left\{(p, \lambda) \in C: f^{k}(p, \lambda)=p, \text { for some } k \geq 1\right\}
$$

be the set of periodic points of $f$. For a point $(p, \lambda) \in P$, let

$$
\begin{aligned}
\Lambda(p, \lambda)=\{\mu: & \mu \text { is an eigenvalue of } D_{p}^{k} f(p, \lambda), \\
& \text { where } k \text { is the minimum period of }(p, \lambda)\} .
\end{aligned}
$$

We call the elements of $\Lambda(p, \lambda)$ the "eigenvalues of $(p, \lambda)$ ".

As in [YA] we assume that $f$ satisfies the following hypotheses:

(H1) All periodic orbits in $f_{0}(C) \cap C$ are attractors. In particular, $f_{0}(C) \cap C$ may be empty.

(H2) There are no periodic orbits of $f$ on $\partial C$ (the boundary of $C$ ).

(H3) If $(p, 1) \in P$, then there is exactly one eigenvalue $\mu \in \Lambda(p, 1)$ such that $|\mu|>1$. The remaining eigenvalues of $(p, 1)$ satisfy $|\mu|<1$.

In addition, we assume an area contracting hypothesis:

(AC) There is a $\theta<1$ such that whenever $(p, \lambda) \in P$ and $\Lambda(p, \lambda)=\left\{\mu_{1}, \ldots, \mu_{n}\right\}$, then $\left|\mu_{i} \mu_{j}\right|<\theta$, for $i \neq j, 1 \leq i, j \leq n$.

Notice that (AC) is satisfied for maps $F$ on $R^{n}$ if $D F$ contracts areas on every two-dimensional subspace.

We mention two examples of maps which satisfy (H1)-(H3) and (AC):

(1) maps which form horseshoes, i.e., where $f_{0}(C)$ is disjoint from $C$ and $f_{1}$ is a full horseshoe map (in the sense of Smale [S]);

(2) area contracting homotopies $f_{\lambda}: D^{n} \rightarrow D^{n}$ such that $f_{0}$ has a single attractor and $f_{1}$ has only unstable, hyperbolic periodic orbits.

Let $O(f)$ be the "orbit space" of $f$; that is, we identify points $(p, \lambda)$ and $(q, \lambda)$ in $P$ if $f^{m}(p, \lambda)=q$, for some $m \geq 1$, and let $O(f)$ be the set of equivalence classes of periodic points under this identification. For ease of notation, we write $(p, \lambda) \in \mathcal{O}(f)$ for any representative $(p, \lambda)$ of an orbit.

Under assumption (AC), each orbit $(p, \lambda)$ in $O(f)$ is in one of the following five disjoint subsets, classified according to the location of the eigenvalues in $\Lambda(p, \lambda)$ :

(1) the set $A$ of attracting orbits $(|\mu|<1$ for all $\mu$ in $\Lambda(p, \lambda))$;

(2) the set $M$ of Möbius orbits $(\mu \in(-\infty,-1)$ for some $\mu$ in $\Lambda(p, \lambda))$;

(3) the set $S$ of saddle orbits $(\mu \in(1, \infty)$ for some $\mu$ in $\Lambda(p, \lambda))$;

(4) $B_{+}=\{(p, \lambda) \in \mathcal{O}(f): 1 \in \Lambda(p, \lambda)\}$; and

(5) $B_{-}=\{(p, \lambda) \in O(f):-1 \in \Lambda(p, \lambda)\}$.

Notice that in each of the cases (2)-(5), at most one eigenvalue in $\Lambda(p, \lambda)$ can lie on or outside the unit circle in the complex plane. We sometimes specify the map for clarity by writing $A(f)$ or $B_{+}(f)$, etc. 
We analyze the structure of path components of orbits for a particular generic class $K$ of maps ${ }^{2}$ in $C^{3}\left(C \times I, R^{n}\right)$. Restricting $K$ to maps which satisfy (AC), then $g$ is in $K$ if all orbits of $g$ are in $S, M$, or $A$, or are one of the following two types of bifurcation orbits:

(1) Orbits in $B_{+}(g)$ are "saddle-node" bifurcation orbits from which two branches of non-Möbius orbits emanate - under (AC), one branch consists of saddles and the other, of attractors. Taken together, these two branches form a local (1dimensional) path of orbits (see precise meaning below) through the saddle node. All orbits on this path near (and including) the saddle node have the same minimum period. Passing through the bifurcation orbit, the path of one eigenvalue in $\Lambda(p, \lambda)$ crosses +1 (with nonzero derivative).

(2) Orbits in $B_{-}(g)$ are "period-doubling" bifurcation orbits from which three branches of orbits - two non-Möbius and one Möbius-emanate. Orbits on one of the non-Möbius branches have twice the minimum period of the bifurcation orbit, while orbits on the other two branches have the same minimum period. Following the low-period path of orbits through the bifurcation orbit, the path of one eigenvalue in $\Lambda(p, \lambda)$ crosses -1 . It was shown in [YA] that under (AC) there are only our possibilities for period-doubling bifurcations; they are pictured schematically below. In these figures, each point represents a periodic orbit in the parametrized orbit space (i.e., a point in $O(g)$ ). The parameter $\lambda$ increases from left to right. The upper branch represents the branch of double period orbits.
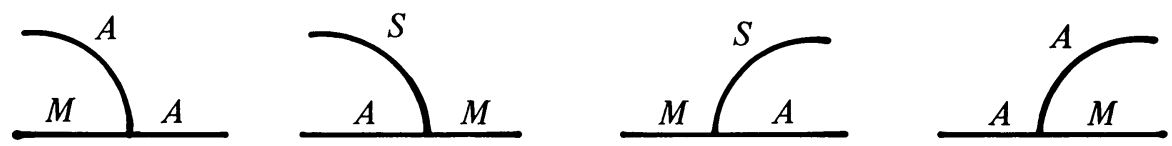

$$
\begin{aligned}
A & =\text { branch of Attractors } \\
M & =\text { branch of Möbius orbits } \\
S & =\text { branch of Saddles }
\end{aligned}
$$

Notice, in particular, that one low-period branch must be orbits in $A$; the other, orbits in $M$.

Let

$$
S=\{(p, \lambda) \in \mathcal{O}(g):(p, \lambda) \notin M(g)\}
$$

be the set of non-Möbius orbits of $g$. By examining the generic bifurcations, it can be seen that each orbit in $S$ lies on a local (1-dimensional) path of orbits in $S$. (By ignoring Möbius orbits, one of the three branches emanating from an orbit in $B_{-}(g)$ is eliminated. The other two non-Möbius branches form a local path through the bifurcation orbit.) We use the term "local path" through an orbit $(p, \lambda)$ to mean the following: there exists a bound $P>0$ and a neighborhood $W$ (in $(x, \lambda)$-space) such that the only orbits within $W$ of period less than $P$ are on the path. The underlying idea of [YA] was to use the orbit (or $\varphi$-) index (as introduced in [MY], [CMY], and [AMY]) to orient these paths of non-Möbius orbits (called "snakes" in $[\mathbf{Y A}]$ ). It was then shown, by following these snakes according to their required

\footnotetext{
${ }^{2}$ Based on techniques of Brunovsky and Peixoto, it was shown in [AMY] that the set $K$ is residual in $C^{3}\left(C \times I, R^{n}\right)$.
} 
orientation, that any snake that starts with a saddle at $\lambda=1$ must terminate in a cascade of attractors.

Here we use similar techniques: We distinguish particular subsets of $O(g)$ to obtain components of orbits which are local paths, and then orient these paths to obtain the results.

THEOREM 1. Let $f$ satisfy (H1)-(H3) and the area contracting hypothesis ( $\mathrm{AC})$. For any orbit $(p, 1)$ of minimum period $k$, the component of $O(f)$ containing $(p, 1)$ has attracting periodic points whose (minimum) period is $2^{m} k$, for each $m \geq 0$.

PROOF. For $(p, 1)$ a saddle, the result is obtained in [YA] (Theorem A). For $(p, 1)$ a Möbius orbit, we give the argument in the generic case only (i.e., for $g \in K$ near $f$ in the $C^{1}$ topology). The limit arguments, which then give the result in the general $C^{1}$ case, are the same as those given in the proof of Theorem $\mathrm{A}$ in [YA]. We assume that $g$ satisfies $(\mathrm{H} 1)-(\mathrm{H} 3)$ and $(A C)$, and that $g_{0}=f_{0}$ and $g_{1}=f_{1}$. (Both $f_{0}$ and $f_{1}$ are generic.) Arguments for these assumptions are given in [YA].

We begin by distinguishing a particular subset of $O(g)$, so as to eliminate one of the three branches of orbits at period-doubling bifurcations.

Let

$$
\begin{array}{r}
\not 1=\{(p, \lambda) \in \mathcal{O}(g):(p, \lambda) \text { is a Möbius orbit of period } k, \text { or }(p, \lambda) \text { is a saddle } \\
\text { or attractor of period } j, \text { for any } j \geq 2 k\} .
\end{array}
$$

By checking each generic bifurcation, it is easily seen that any orbits in $\not$ lies on a local path of orbits in $\nLeftarrow \cup B_{+}(g) \cup B_{-}(g)$.

Now let $(p, 1)$ be a Möbius orbit of period $k$, and let $\Gamma$ be the path of orbits in $\forall \cup B_{+} \cup B_{-}$containing $(p, 1)$. We orient $\Gamma$ according to the following scheme,

$$
\vec{A}, \quad \overleftarrow{M}, \quad \overleftarrow{S}
$$

(The arrows above $A, M$, and $S$, respectively, indicate whether $\Gamma$ should be followed to the right $(\rightarrow)$ through increasing $\lambda$ values, or to the left $(\leftarrow)$ through decreasing $\lambda$ values, at each point $(p, \lambda)$ on $\Gamma$ in $A, M$, or $S$.) Notice that a local path of nonMöbius orbits in $\nvdash \cup B_{+} \cup B_{-}$can only change direction (in $\lambda$ ) at a saddle-node or period-doubling bifurcation. At such a point, the direction changes if and only if orbits along the path go from attractors to saddles (or vice versa). Each Möbius orbit of $g$ lies on a path (in $\lambda$ ) of Möbius orbits whose endpoints are orbits in $B_{-}(g)$. In particular, under (AC) the paths of Möbius orbits do not change direction in $\lambda$. Hence, by again checking each generic bifurcation, this orientation is seen to be well defined on $\Gamma$.

Starting at $(p, 1)$, we follow $\Gamma$ to the left, as its orientation requires. By (H2), $\Gamma$ cannot intersect $\partial C$. By $(\mathrm{H} 1), \Gamma$ cannot intersect $C \times\{0\}$, since its direction of approach would imply that $g_{0}$ has either saddles, Möbius orbits, or bifurcation orbits-all of which are impossible. Also, $\Gamma$ cannot re-intersect $C \times\{1\}$, since its direction of approach would imply that $f_{1}$ has either attractors or bifurcation orbits-both of which are impossible by (H3). Hence, $\Gamma$ has exactly one endpoint (at $\lambda=1$ ). We argue that the periods of orbits on $\Gamma$ go to infinity. (This is essentially the proof of Proposition 3.5 in [YA]. We include it for the reader's convenience.) The generic family $\Gamma$ consists of segments of orbits in $A, M$, or $S$, joined by bifurcation orbits in $B_{+}$or $B_{-}$. Suppose there are only a finite 
number of bifurcation orbits. Then $\Gamma$ will be the union of a finite number of these segments together with the bifurcation orbits, and thus will have two endpoints, a contradiction. Hence, $\Gamma$ must contain an infinite number of bifurcation orbits. If the periods of the bifurcation orbits are bounded, then some sequence $\left\{b_{n}\right\}_{n \in N}$ of these orbits converges to an orbit $\beta$ of $g$. However, it is easily seen that the orbit $\beta$ is not of generic type. Hence the periods of the bifurcation orbits on $\Gamma$ go to infinity, although not necessarily monotonically. In order for these periods to go to infinity, $\Gamma$ must contain orbits in $B_{-}(g)$ of period $2^{m} k$, for each integer $m, m \geq 0$. Since each such orbit must be approached through attractors (for $m \geq 1$ ), $\Gamma$ contains an attractor of period $2^{m} k$, for each $m \geq 1$.

To obtain an attractor of period $k$ on the same orbit component as $(p, 1)$, simply notice that the first orbit in $B_{-}(g)$ encountered as $\Gamma$ is followed from $(p, 1)$ will necessarily have a branch of attractors of period $k$ emanating from it.

THEOREM 2. Let $f$ satisfy (H1)-(H3) and (AC). For each saddle at $\lambda=1$ or attractor at $\lambda=0$ having period $k$, there is a sequence $\left\{q_{i}\right\}_{i \in N}$ of Möbius orbits at $\lambda=1$ such that the period of $q_{i}$ is $2^{i} k$ for each $i \geq 0$. Two such sequences are identical or disjoint, and every orbit is in such a sequence. Hence the orbits at $\lambda=0$ and $\lambda=1$ are partitioned into period-doubling sequences. Furthermore, an entire sequence lies in one component of orbits of $f$.

ProOF. Again, we begin by showing the result in the generic case, for $g \in K$ satisfying (H1)-(H3) and (AC). Since we assume that $g_{0}=f_{0}$ and $g_{1}=f_{1}$, the only statement that requires limit arguments for the general $C^{1}$ case is the last.

As in the proof of Theorem 1, we distinguish a particular subset of $O(g)$ : For $n$ even, let

$$
\begin{aligned}
P_{n}=\{(p, \lambda) \in \mathcal{O}(g): & (p, \lambda) \text { is in } A \text { or } S \text { and has period } n ; \\
& \text { or }(p, \lambda) \text { is in } M \text { and has period } n \text { or } n / 2\} .
\end{aligned}
$$

For $n$ odd, omit Möbius orbits of period $n / 2$ from $P_{n}$.

Again, it is easily checked that any orbit in $P_{n}$ lies on a local path of orbits in $\rho_{n} \cup B_{+} \cup B_{-}$.

We orient these paths as follows:

$$
\text { period } n: \vec{A}, \overleftarrow{S}, \vec{M}, \quad \text { period } n / 2: \overleftarrow{M}
$$

We call such an oriented path of orbits in $P_{n} \cup B_{+} \cup B_{-}$an n-path. Basically, we are trying to follow a path of orbits of period $n$-the only possible obstacle is the occurrence of a "period-halving" bifurcation along the path, at which point two branches of orbits emanating from the bifurcation orbit (in $B_{-}(g)$ ) have period $n / 2$. In this case we follow the low-period Möbius branch.

Recognizing that orbits on $n$-paths have bounded periods $(n$ or $n / 2)$ and following the proof of Theorem 1 , it must be the case that each $n$-path must have two endpoints on the boundary of $C \times I$. At least one of these endpoints must occur at $\lambda=1$.

Let $(p, 1)$ be a saddle of period $k($ at $\lambda=1)$. Then $(p, 1)$ lies on a $k$-path which necessarily re-intersects $C \times\{1\}$ as a Möbius orbit $\left(q_{0}, 1\right)$ of period $k$. (Following the orientation on the $k$-path, the only other possibilities are either an attractor of 
period $k$ or a bifurcation orbit at $\lambda=1$, or a Möbius orbit of period $k / 2$ at $\lambda=0$ all of which are impossible.) If $(p, 0)$ is an attractor of period $k$ at $\lambda=0$, then the same argument holds, resulting in a Möbius orbit $\left(q_{0}, 1\right)$ of period $k($ at $\lambda=1)$. Now, starting with the Möbius orbit $\left(q_{0}, 1\right)$, we follow a $(2 k)$-path and return to $C \times\{1\}$ at a Möbius orbit $\left(q_{1}, 1\right)$ of period $2 k$. Continuing in this manner, we obtain a sequence $\left\{q_{i}, 1\right\}_{i \geq 0}$ of Möbius orbits such that $\left(q_{i}, 1\right)$ has period $2^{i} k$ and is contained in the same component of $\mathcal{O}(g)$ as the saddle $(p, 1)$ (or attractor $(p, 0))$. In addition, since this process is reversible, we can start with any Möbius orbit of period $2^{i} k$, for some $i \geq 0$, and trace it back through Möbius orbits of periods $2^{j} k(0 \leq j<i)$ to a saddle of period $k$ at $\lambda=1$ (or an attractor at $\lambda=0$ ). We call the sequence of orbits obtained in this manner a $k$-sequence; i.e., $\left\{p_{i}\right\}_{i \geq 0}$ is a $k$-sequence if (1) $p_{0}$ is a saddle of period $k$ at $\lambda=1$ (or an attractor at $\lambda=0$ ); (2) $p_{i}$, for $i \geq 1$, is a Möbius orbit of period $2^{i-1} k$ at $\lambda=1$; and (3) $p_{i-1}$ and $p_{i}$ are endpoints of some $(i k)$-path, for each $i \geq 1$.

Each orbit at $\lambda=0$ and $\lambda=1$ is in a $k$-sequence, for some $k \geq 1$. The process described (i.e., following $n$-paths) defines a map from a saddle (or attractor) of period $k$ to a Möbius orbit of period $k$ and from a Möbius orbit of period $k$ to a Möbius orbit of period $2 k$. Each orbit has a unique inverse under this mapping. (Since $k$-paths are disjoint, the inverse of a Möbius orbit of period $k$ is either a Möbius orbit of period $k / 2$ or a saddle (or attractor) of period $k$, not both. Hence no orbit is in more than one $k$-sequence, for any $k \geq 1$, and a partition is obtained.

Finally, to see that an entire $k$-sequence lies on one component of orbits of $f$, we choose a sequence $\left\{g_{m}\right\}_{m \in N}$ of maps in $K$ such that $\lim _{m \rightarrow \infty} g_{m}=f$ (in the $C^{1}$ topology) on the bounded set $C \times I$. Any two orbits in a $k$-sequence are endpoints of a finite sequence of connecting $(i k)$-paths, for $g_{m} \in K$. Taking limits as $g_{m} \rightarrow f$, it follows that this finite sequence of $(i k)$-paths will converge to a connected set of orbits of $f$.

REMARKS. (1) Theorem 2 yields a simple formula which relates the number $M(n)$ of Möbius orbits of minimum period $n$ in the horseshoe to the number $S(i)$ of saddles of minimum period $i, i \leq n$; namely,

$$
M\left(2^{n} k\right)=\sum_{j=0}^{n} S\left(2^{j} k\right),
$$

for any $n \geq 0$ and $k=1,3,5, \ldots$.

(2) Theorem 2 may seem straightforward when looking at the simplest kinds of cascades. However, the idea of $k$-paths is essential here, since if we merely follow a snake (i.e., path of non-Möbius orbits) from a saddle at $\lambda=1$ (as in [YA]), the periods of orbits along the snake do not necessarily increase monotonically. They may decrease occasionally, throwing off many paths of low-period Möbius orbits.

\section{REFERENCES}

[AMY] K. T. Alligood, J. Mallet-Paret and J. A. Yorke, An index for the global continuation of relatively isolated sets of periodic orbits, Geometric Dynamics, Lecture Notes in Math., vol. 1007, Springer-Verlag, New York and Berlin, pp. 1-21.

[CMY] S. N. Chow, J. Mallet-Paret and J. Yorke, A bifurcation invariant: degenerate orbits treated as clusters of simple orbits, Geometric Dynamics, Lecture Notes in Math., vol. 1007, SpringerVerlag, Berlin and New York. 
[F] J. Franks, Period doubling and the Lefschetz formula, Trans. Amer. Math. Soc. 287 (1985), 275283.

[MY] J. Mallet-Paret and J. A. Yorke, Snakes: oriented families of periodic orbits, their sources, sinks, and continuation, J. Differential Equations 43 (1982), 419-450.

[S] S. Smale, Differentiable dymamical systems, Bull. Amer. Math. Soc. 73 (1967), 747-817.

[YA] J. A. Yorke and K. T. Alligood, Period doubling cascades of attractors: a prerequisite for horseshoes, Comm. Math. Phys. (to appear).

Department of Mathematics, College of Charleston, Charleston, South CAROLINA 29424

Current address: Department of Mathematics, George Mason University, Fairfax, Virginia 22030 